\title{
Noise Reduction of Seismic Signal via Empirical Mode Decomposition
}

\author{
Baotong Liu \\ School of Electronic Information and Electrical Engineering, Tianshui Normal University, China \\ liubt163@163.com
}

Keywords: Seismic signal, Denoising, Predictive filter, Intrinsic mode.

\begin{abstract}
This paper developed a denoising method termed $f$ - $x$ empirical-mode decomposition (EMD) predictive filtering. In this new method, we first applied EMD to each frequency slice in the $f-x$ domain and obtained several intrinsic mode functions (IMFs). Then, an autoregressive model was applied to the sum of the first few IMFs to predict the useful steeper events. Finally, the predicted events were added to the sum of the remaining IMFs. This process improved the prediction precision by using an EMD-based dip filter to reduce the dip components before $f$ - $x$ predictive filtering. A synthetic data example is provided to show the performance of presented method.
\end{abstract}

\section{Introduction}

Random noise attenuation played an important role in seismic signal processing. Canales (1984) first uses $f-x$ predictive filtering to attenuate random noise [1]. Since then, continuous efforts have been made to improve the predictive precision or to modify the conventional version to meet better the requirements set by various applications [2][3]. When the subsurface is extremely complex, $f-x$ predictive filtering doesn't yield good results because of the large number of dip components that need to be predicted.

Huang et al. propose a new signal processing method that uses empirical mode decomposition (EMD) to prepare stable input for the Hilbert transform. The essence of EMD is to stabilize a nonstationary signal. That is, to decompose a signal into a series of intrinsic mode functions (IMFs) [4]. Each IMF has a relatively local constant frequency. The frequency of each IMF decreases according to the separation sequence of each IMF. EMD is a breakthrough in the analysis of linear and stable spectra. It adaptively separates nonlinear and nonstationary signals, which are features of seismic data, into different frequency ranges. Bekara and van der Baan apply $f-x$ EMD to attenuation of random and coherent noise with good results [5]. For the purpose of random noise attenuation, the $f-x$ domain EMD method can only be applied on NMO-corrected or poststack seismic data. With profiles containing dipping events, these methods will suppress some of the useful energy.

In this paper, we present a new approach, termed $f-x$ empirical mode decomposition predictive filtering (EMDPF) that combines $f-x$ EMD and $f-x$ predictive filtering. This new noise attenuation methodology can adapt to more complex seismic profiles than $f-x$ EMD, and preserve more useful energy than $f-x$ predictive filtering. The $f-x$ EMDPF uses an EMD-based dip filter to reduce the dip components for the subsequent predictive filtering to improve the predictive precision.

\section{Predictive Filtering in Frequency-Space Domain}

Let $s(t, h)(h=1,2, \ldots, H)$ be the signal of trace and $h$ and $H$ be the number of traces. If the slope of a linear event with constant amplitude in a seismic section is $\lambda$, then

$$
s(t, h+1)=s(t-h \lambda \Delta x, 1)
$$

where $\Delta x$ denotes the trace interval. Eq. 1 an be transformed into the frequency domain to give

$$
s(f, h+1)=s(f, 1) e^{-i 2 \pi f h \lambda \Delta x} .
$$


For a specific frequency $f_{0}$, from Eq. 2, we can obtain a linear recursion that is given by

$$
s\left(f_{0}, h+1\right)=a\left(f_{0}, 1\right) s\left(f_{0}, h\right),
$$

where $a\left(f_{0}, 1\right)=e^{-i 2 \pi f_{0} \lambda \Delta x}$. This recursion is a first order difference equation, also known as an autoregressive (AR) model of order 1. Similarly, superposition of $p$ linear events in the $t-x$ domain can be represented by an AR model of order $p$ as the following equation:

$$
s\left(f_{0}, h+1\right)=a\left(f_{0}, 1\right) s\left(f_{0}, h\right)+a\left(f_{0}, 2\right) s\left(f_{0}, h-1\right)+\cdots+a\left(f_{0}, p\right) s\left(f_{0}, h+1-p\right),
$$

where $a\left(f_{0}, h\right)(h=1,2, \cdots, p)$ denotes the predictive error filter, with a length of $p$. The prediction error energy $E\left(f_{0}\right)$ is given by the following equation:

$$
E\left(f_{0}\right)=\left\|a\left(f_{0}, h\right) * S\left(f_{0}, h\right)-S\left(f_{0}, h+1\right)\right\|_{2}^{2},
$$

where the symbol * denotes convolution and \|\|$_{2}^{2}$ denotes the least-squares energy. By minimizing the prediction error energy $E\left(f_{0}\right)$, we can get the filtering operator $a\left(f_{0}, m\right)$. Applying this operator to the spatial trace yields the denoised results for the frequency slice $f_{0}$.

The $f-x$ predictive filtering works perfectly on a single event. However, when the number of different dips is increased, the seismic section becomes more complex and predictive filtering is not as effective. In particular, when the number of dips is extremely large, as occurs with hyperbolic events, $f-x$ predictive filtering fails to achieve acceptable results. If we can first reduce the number of dips, then by applying the same $f-x$ predictive filtering, the predictive precision will improve. That is the subject of the section on improved denoising method via EMD predictive filtering.

\section{Empirical Mode Decomposition}

Provided that $s(t), c_{n}(t), r(t)$, and $N$ denote the original nonstationary signal, the separated IMFs, the residual, and the number of IMFs, respectively, the mathematical principle of EMD can be expressed as

$$
s(t)=\sum_{n=1}^{N} c_{n}(t)+r(t) .
$$

For a nonstationary signal $s(t)$, using Eq. 6, we get a finite set of subsignals $c_{n}(t),(n=1,2, \cdots, N)[4]$.

Because of the frequency decreasing property, EMD has been used outside geophysics for noise attenuation. Because random noise represents mainly the high-frequency components, by removing the IMFs with the highest frequency, we can attenuate this type of noise. However, in exploration geophysics, applying EMD to time traces is not effective because of the mode mixing problem [6].

Instead of $t-x$ domain EMD, a $f-x$ domain EMD method to attenuate random noise in seismic data has been proposed by Bekara and van der Baan [5]. They applying EMD on each frequency slice in the $\mathrm{f}-\mathrm{x}$ domain, and suppress the higher wavenumber components that mainly represent random noise. However, a problem occurs when applying $f-x$ EMD because the high-wavenumber dipping events will also be removed. This problem occurs because, for many data sets, the random noise and any steeply dipping coherent energy make a significantly larger contribution to the high wavenumber energy in the $f-x$ domain than any desired signal. The detailed algorithm steps of $f-x$ EMD are given in [5]. 


\section{Improved Denoising Method via EMD Predictive Filtering}

The $f$ - $x$ empirical mode decomposition predictive filtering (EMDPF) uses the property that the first few (generally 1-3) IMFs for each frequency slice in the $f-x$ domain contain the high dip angle components and random noise. Thus, the leaked dipping events can be obtained by applying a predictive filter to these IFMs. Adding the predicted signal to the sum of the remaining IMFs will suppress random noise without harming the effective signals.

The detailed algorithmic steps of $f-x$ EMDPF are shown below:

1) Select a time window and transform the data to the $f-x$ domain.

2) For every frequency,

(1) separate real and imaginary parts in the spatial sequence,

(2)compute IMF1, for the real signal and subtract it to obtain the filtered real signal,

(3)apply an AR model to IMF1 and add the result to the sum of the remaining IMFs,

(4)repeat for the imaginary part, and

(5)combine to create the filtered complex signal.

3) Transform data back to the $t-x$ domain.

4) Repeat for the next time window.

It should be emphasized that the number of the filtered IMFs is not limited to one, but is selected according to the noise level and the distribution of the dip components within the specific seismic data set. Generally, the number of IMFs for filtering is within the range of 1-3.

\section{Numerical Experiment}

Fig. 1a is a noise free data, Fig. 1b is Gaussian white noise, and Fig. 1c is the noisy data. Fig. 1g, 1h, and $1 \mathrm{i}$ show the removed noise sections corresponding to $f-x$ predictive filtering, $f-x$ EMD, and EMDPF, respectively. From Fig. 1g, we can see that $f-x$ predictive filtering harms flat and dipping events to some extent. Although by increasing the length of the predictive step, we can decrease the damage done to the signals, the noise suppression is less effective because of the stronger prediction of noise. Also, as seen in Fig. 1d, the $f-x$ predictive filtering introduces some artifacts. In Fig. 1h, we see that $f-x$ EMD tends to harm much of the dip energy but preserves entirely the flat events. Using the same predictive filtering parameters, we see from Fig. 1i that flat and dipping signals are hardly affected when $f-x$ EMDPF is applied. In this example, the first IMF is removed for prediction in the process of $f-x$ EMDPF.
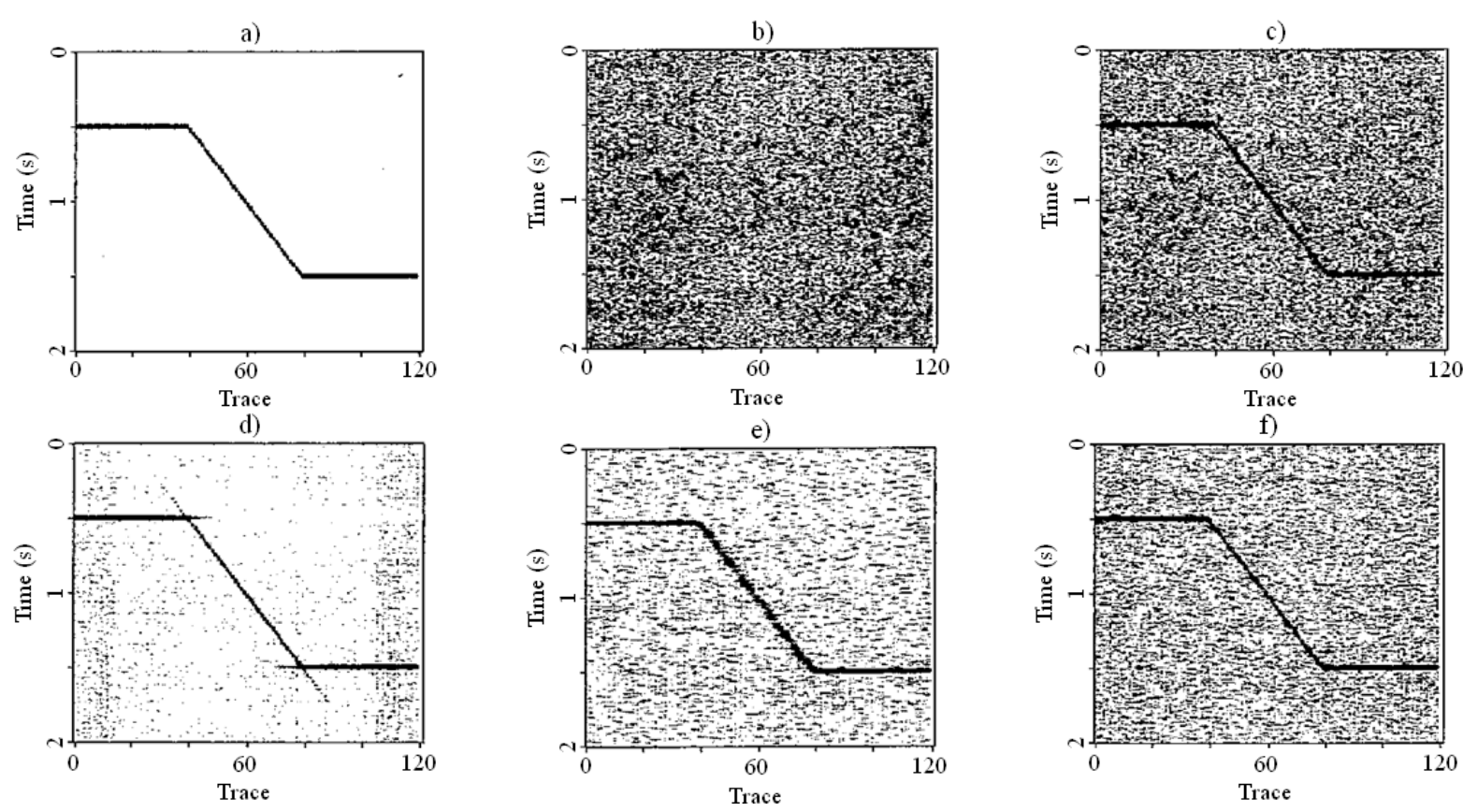
g)

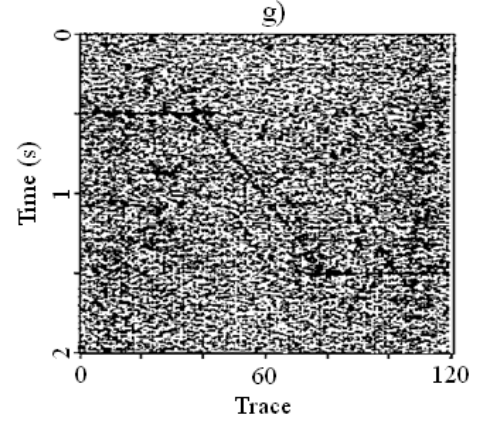

h)

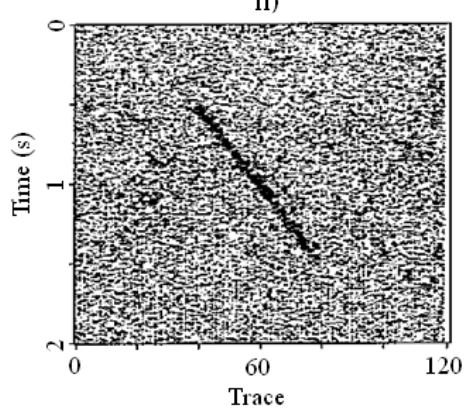

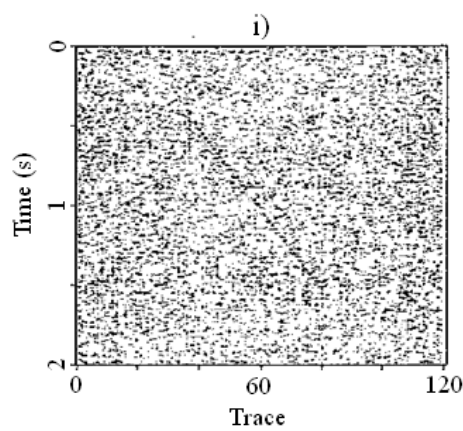

Figure 1. Comparison of denoising effects. a)Clean data, b)Gaussian white noise, c)noisy data, d)denoised result by $f-x$ predictive filtering. e) denoised result by $f-x$ EMD, f)denoised result by $f-x$ EMDPF, g)removed noise section corresponding to d), h) removed noise section corresponding to e), and i) removed noise section corresponding to f).

\section{Conclusions}

This paper has presented a new denoising method suitable for complex subsurface structures. By using the AR model to predict the steeply dipping event, $f-x$ EMDPF can deal with complex seismic profiles that conventional $f-x$ EMD cannot candle. By applying an EMD-based adaptive dip filtering in advance, $f-x$ EMDPF can preserve more useful energy as compared with conventional $f$ - $x$ predictive filtering.

\section{Acknowledgement}

This work was financially supported by National Natural Science Foundation of China under Grant No. 41264002.

\section{References}

[1] L. Canales: SEG Expanded Abstracts, 54 ${ }^{\text {th }}$ Annual International Meeting (1984): p. 525

[2] J. Guo, X. Zhou, and H. Yang: Oil Geophysical Prospecting Vol. 30 (1995), p. 207

[3] Y. Kang, C. Yu, W. Jia, and C. Wang: Oil Geophysical Prospecting Vol. 38 (2003), p. 136

[4] Shen, S. R. Long, et. al.: Proceeding of the Royal Society of London Series A, 454 (1998), p. 136

[5] M. Bekara, M. van der Baan: Geophysics Vol. 74 (2009), p. V89

[6] M. Copecky: Acta Polytechnica Vol. 50 (2010), p. V49 